of the paragraph had communicated with myself previously to "rushing into print," he would have received such information as would have prevented him giving publicity to a statement which however literally correct, is errone sus in essence.

EDWARD HULL,

Director of the Geological Survey of Ireland

Geological Survey of Ireland, Dublin, November 6

[We were indebted for the note to the courtesy of Mr. Kinahan, of the Geological Survey of Ireland.-ED.]

\section{Lunar Ring}

WHILE experimenting on the actenic power of lunar light on August 30 last (period of full moon), at 9.30 P.M., I obtained, with a minute-and-a-half exposure, a photographic negative of the moon, which shows a distinct and well-defined ring or glory around it which was not visible to the naked eye on looking directly at the moon in a clear and cloudless sky, nor was there any halo on the ground glass of the camera, nor on the lens, at the time of observation This is a copy of it from the negative.

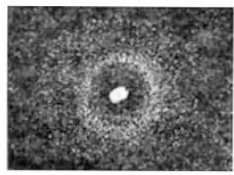

I used no clock-work arrangement with the camera, but allowed the moon to traverse the plate, and I have since then taken several photographic observations under various conditions. I have taken the moon in all her phases, with long and short exposures, in clear and cloudless sky, and never could get a ring even faintly defined. I have also heated the camera and screwed the cold lens into it, carried it into a colder atmosphere in order to produce condensation of dew. I have placed two small separate openings in front of the lens; on one occasion I dusted puff-ball spores upon the lens; on another I breathed warm breath upon it, but never got anything but decided burr, which was always densest near the limb of the moon and gradually tapered away towards the circumference like a bright light seen through a thick fog, but no appearance of ring. I have also taken observations when scud was passing rapidly over the moon, when perfect prismatic halos were visible to the naked eye, but no ring was ever impressed on the photographs; nothing more than a haze, such as that produced by breathing on the lens. The next full moon (September 29) was totally obscured, so that I failed to get an observation then; but last evening (October 29), at Io 1'M. I I was fortunate to get one fine, clear exposure of one-and-a half minute, and was pleased to see a clear and well-defined ring rise up on the plate during development, similar in every respect to that obtained on August 29, showing clearly that this unusual appearance is dependent upon the position of the moon in her orbit, she being in opposition when she manifests ring-giving power and shows us a crown. But why is this? What is the cause of this unusual, and, I believe, hitherto undescribed, appearance? Why should this ring be invisible to the naked eye and yet give a luminous impression on a photographic plate? Why should it appear only at full moon period and not at any other phase? Can it have any connection with what Mr. Newall saw round Mars through his huge telescope? If the moon had an atmosphere similar to that of the earth, and a star of some magnitude were occulted by the moon at that particular time, it is possible that its light in passing through the lunar atmosphere might be refracted so as to show a corona round the moon; but it is pretty generally acknowledged that there is no atmosphere surrounding it, therefore there can be no refraction.

It might be that the solar rays in passing through the upper regions of the earth's atmosphere are so defracted that the ultraviolet rays (though invisible) are thus rendered visible.

It is also possible that the doubly-reflected lunar light (the ashy light), in passing back to the moon from the earth, encounters on its passage the reflected solar rays from the moon, arresting and nullifying in proportion to its strength, so much of the light proceeding from the moon thereby causing a clear space around the moon-limb, a region of inertia, while the reflection from the disk of the earth, being larger than the moon's reflecting disk, will show itself as a ring on the outer edge of the neutral zone, much in the same manner as two heliographic reflectors would act if they were so arranged as to throw their respective reflections directly into and upon each other, the one being small and the other larger, just as the moon is the smaller and the earth the larger body, the smaller body reflecting a smaller, brighter light, while the larger body would reflect from its broader disk a less brilliant light with a feebler force, yet not so feeble as to prevent it arresting an amount of force equal to itself.

Sunderland, October 30

George BerWick

[Dr. Berwick's explanation appears scarcely sound for it involves the assumption that a ray of light meeting another can arrest it; and also it involves the visibility of such rays while traversing space. We would remark that faint halos due to atmospheric causes are often seen almost masked by the brightness of a full moon, and the photograph being over-exposed, so far as the moon is concerned, does not show the relative actinic brightness of moon and halo. Would Dr. Berwick try further exferiments with shorter exposures, and also ascertain from a number of photographs how far on either side of full moon a halo can be photographed, and whether it is always present during similar periods?-ED.]

\section{Phosphorescence}

A FEW days ago my attention was drawn to the phosphorescence of some fish (haddock) just received from the coast. The light was most brilliant about the fins and inside of the fish, which had been gutted. A spectroscope of low dispersive power showed all the light to belong to the green part of the spectrum. Approximate measures yave $557^{\circ} 5\left(\mathrm{~mm}\right.$.) and $4^{\mathrm{SS}} \mathrm{S}^{\circ} 4$ as the extreme wavelengths, the part from 557.5 to 503.4 being somewhat brighter than the remainder, with a feebly indicated maximum at 527.6 .

In the hope of getting a brighter spectrum the fish were washed in as small a quantity of water as possible. This water became highly phosphorescent, and when agitated in a bowl, gave beautiful luminous caustics, but neither in the bowl nor in a glass trough, nor in a tube of half-inch bore, did the liquid give a brighter spectrum than that afforded by the fish.

A large bubble of air was inclosed with the liquid in the tube. When the tube was violently agitated, it became luminous from end to end ; if then held vertically, the light rapidly faded except near the top of the liquid, but on suddenly inverting the tube, the bubble of air slowly ascended, causing the whole contents of the tube to phosphoresce very brilliantly. This was a most striking phenomenon. After the lapse of some nine hours, the liquid had almost entirely lost the power of giving light.

The Observatory, Dunecht, Aberdeen, November 5

RALPH COPELAND

\section{The "False Dawn"}

FOR some time past certain considerations had led me gradually to infer that the "False Dawn" of the very extensive literature of Islām, whether Arabic, Persian, or Turkisb, \&c., and whether prose or verse, is another name for the "Zodiacal Light." No dictionary yet published so explains it.

I submitted my ideas and reasons to a number of English and foreign astronomers and linguists. All expressed their concurrence in those views; but direct proof of their correctness was not at once forthcoming. Recently, however, through the kindness of the Hydrographer to the Admiralty, a most obliging effort was made to solve this question by Capt. Wharton, commanding H.M.S. Faren, now cruising in the Sea of Marmora. The method employed by that officer, and its conclusive result, cannot be better described than by giving his own words as follows :-

$$
\text { H.M.S. Fown, Tuzla Bay, }
$$$$
\text { September 26, } 1879
$$

\section{Dear Capt. Evans,}

For the information of Mr. Redhouse, I have to tell you that I can satisfactorily answer his question as to the false dawn of the Turks.

On the morning of the 20 th instant, at $3.30 \mathrm{~A} . \mathrm{M}$., I went to a mosque at Buyukdere, ard interviewed the Imaum, who, on being asked for the "fejri kyazib," I at once pointed out the zodiacal light, then brightly shining in the east. . . . There can be no doubt as to the coincidence of the two.

$$
\text { Yours sincerely, }
$$
W. J. L. Wharton

I "Fejri Kyazib" is the Arabic express on for "the false dawn." 